\title{
Long-term safety and efficacy of adalimumab for intestinal Behçet's disease in the open label study following a phase 3 clinical trial
}

\author{
Nagamu Inoue ${ }^{1}$, Kiyonori Kobayashi ${ }^{2}$, Makoto Naganuma ${ }^{3}$, Fumihito Hirai ${ }^{4}$, Morio Ozawa ${ }^{5}$, Dilek Arikan ${ }^{6}$, \\ Bidan Huang ${ }^{6}$, Anne M. Robinson ${ }^{6}$, Roopal B. Thakkar ${ }^{6}$, Toshifumi Hibi ${ }^{7}$ \\ ${ }^{1}$ Center for Preventive Medicine, Keio University Hospital, Tokyo, ${ }^{2}$ Department of Gastroenterology, Kitasato University Hospital, Kanagawa, \\ ${ }^{3}$ Division of Gastroenterology \& Hepatology, Department of Internal Medicine, Keio University School of Medicine, ${ }^{4}$ IBD Center, Fukuoka \\ University Chikushi Hospital, Fukuoka, ${ }^{5}$ AbbVie GK, Tokyo, Japan, ${ }^{6}$ AbbVie Inc., North Chicago, IL, USA, ${ }^{7}$ Center for Advanced IBD Research \\ and Treatment, Kitasato Institute Hospital, Kitasato University, Tokyo, Japan
}

Background/Aims: Intestinal Behçet's disease (BD) is an immune-mediated inflammatory disorder. We followed up the patients and evaluated safety profile and effectiveness of adalimumab for the treatment of intestinal BD through 100 weeks rolled over from the 52 week clinical trial (NCT01243671). Methods: Patients initiated adalimumab therapy at $160 \mathrm{mg}$ at week 0 , followed by $80 \mathrm{mg}$ at week 2, followed by $40 \mathrm{mg}$ every other week until the end of the study. Long-term safety and all adverse events (AEs) were examined. The efficacy was assessed on the basis of marked improvement (MI) and complete remission (CR) using a composite efficacy index, which combined global gastrointestinal symptoms and endoscopic assessments. Results: Twenty patients were enrolled in this study; 15 patients received adalimumab treatment until study completion. The incidence of AEs through week 100 was 544.4 events/100 person-years, which was comparable to the incidence through week 52 (560.4 events/100 person-years). No unexpected trend was observed and adalimumab was well tolerated. At weeks 52 and 100 , $60.0 \%$ and $40.0 \%$ of patients showed MI, respectively, and $20.0 \%$ and $15.0 \%$ of patients showed CR, respectively. Conclusions: This report demonstrates 2 years safety and effectiveness of adalimumab in intestinal BD patients. Patients with intestinal BD refractory to conventional treatment receiving up to 2 years of adalimumab treatment demonstrated safety outcomes consistent with the known profile of adalimumab, and the treatment led to sustained reduction of clinical and endoscopic disease activity. (Intest Res 2017;15:395-401)

Key Words: Biological products; Endoscopy; Ulcer; Behçet’s disease, intestinal

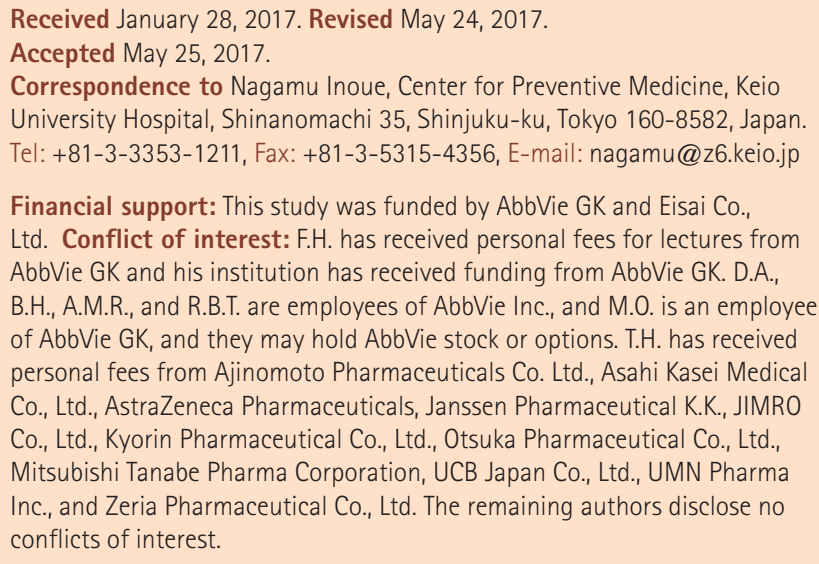

\section{INTRODUCTION}

Behçet's disease (BD) is a multisystem immune-mediated inflammatory disorder characterized by recurrent oral aphthous ulcers, uveitis, skin lesion and genital ulcers. ${ }^{1} \mathrm{BD}$ may also involve visceral organs such as the intestinal tract, nervous system, and vascular system. There have been very few reports regarding their long-term prognosis because these special types of disease are rare and life-threatening in severe case.

Adalimumab has been shown to be effective to several diseases associated with tumor necrosis factor $\alpha$ (TNF- $\alpha)$, and

\footnotetext{
๑ Copyright 2017. Korean Association for the Study of Intestinal Diseases. All rights reserved.

This is an Open Access article distributed under the terms of the Creative Commons Attribution Non-Commercial License (http://creativecommons.org/licenses/by-nc/4.0)

which permits unrestricted non-commercial use, distribution, and reproduction in any medium, provided the original work is properly cited.
} 
has been approved for the treatment of rheumatoid arthritis, psoriatic arthritis, ankylosing spondylitis, CD, psoriasis, juvenile idiopathic arthritis, uveitis, and UC. We previously reported the 52 week results of a phase 3 clinical study demonstrating the efficacy, safety and pharmacokinetics of adalimumab in Japanese intestinal BD patients (clinical trial number: NCT01243671). ${ }^{2}$ Adalimumab was approved in Japan on May 16, 2013 for intestinal BD based on the data from this clinical study. Here we report the long-term (up to 100 weeks) safety and efficacy of adalimumab in intestinal BD patients.

\section{METHODS}

\section{Patients}

This study was conducted at 12 sites in Japan, from November 2010 to June 2013, to evaluate the safety and effectiveness of adalimumab in intestinal BD patients refractory to conventional therapies. ${ }^{2}$ The institutional review board of each center approved the study protocol, the informed consent, and all other forms of patient information related to the

Table 1. Diagnostic Criteria for Behçet's Disease (2003, Study Group of the Health, Labour and Welfare Ministry) ${ }^{3}$

\section{Diagnostic criteria for Behçet's disease}

(1) Major symptoms

(i) Recurrent aphthous ulcers of oral mucosa

(ii) Skin symptoms

(a) Erythema nodosum-like eruption

(b) SC thrombophlebitis

(c) Folliculitis-like eruption and acne-like eruption Reference finding: enhanced skin irritability

(iii) Ocular symptoms

(a) Iridocyclitis

(b) Uveoretinitis (chorioretinopathy)

(c) The following findings, if any, conform to (a) or (b): posterior synechia, lens pigmentation, chorioretinal atrophy, optic atrophy, complicated cataract, secondary glaucoma, and phthisis bulbi considered to have exceeded (a) and (b)

(iv) Genital ulcers

(2) Minor symptoms

(i) Arthritis not accompanied by deformity or rigidity

(ii) Epididymitis

(iii) Gastrointestinal lesions, typically ileocecal ulcers

(iv) Vascular lesions

(v) Moderate or severe lesions of the CNS

(3) Criteria for disease-type diagnosis

(i) Complete type: appearance of 4 major symptoms during the disease course

(ii) Incomplete type

(a) Appearance of 3 major symptoms or 2 major and 2 minor symptoms during the disease course

(b) Appearance of typical ocular symptoms and other 1 major symptom, or 2 minor symptoms during the disease course

(iii) Suspect: appearance of partial major symptoms not satisfying the conditions of abortive type, and recurrence or exacerbation of typical minor symptoms

(iv) Special lesions

(a) Intestinal BD: the presence or absence of abdominal pain and occult blood reaction should be confirmed

(b) Vascular BD: aortic or small-arterial disorders should be differentiated

(c) Neural BD: the presence or absence of headache, paralysis, cerebrospinal disease type, or psychiatric symptoms should be confirmed

BD, Behçet's disease. 
study. The method and entry criteria have been described, ${ }^{2}$ and diagnosis was based on the diagnostic criteria for BD (2003, Study Group of the Health, Labour and Welfare Ministry) (Table 1$)^{3}$

Briefly, patients aged $\geq 15$ years with intestinal BD, an ileocecal ulcer of $\geq 1 \mathrm{~cm}$ in diameter, and a global gastrointestinal (GI) symptom score of $\geq 3$ (affected patient's daily life) were eligible. Global GI symptoms as determined by the patient's daily life status and endoscopic assessment scores as determined by the ulcer size are employed as evaluation criteria (Table 2). A combination of the scores is used to characterize the disease state after treatment.

\section{Study Treatment}

The study protocol was amended in August 2011 to change the treatment period from 52 weeks to allow administration of study drug until the approval of adalimumab for intestinal BD in Japan.

Treatment with adalimumab was initiated at $160 \mathrm{mg}$; after 2 weeks, patients received $80 \mathrm{mg}$, followed by $40 \mathrm{mg}$ every other week until the study end. Patients with inadequate response or disease flare could dose-escalate to $80 \mathrm{mg} \mathrm{ev}$ ery other week (this regimen is preferred in Japan to $40 \mathrm{mg}$ weekly as many patients in Japan prefer to receive injections in the health care provider's setting). Patients who completed the evaluation at week 52 and wished to continue treatment could do so at the investigator's discretion (Fig. 1).

\section{Analysis}

All patients who received $\geq 1$ injection of adalimumab were included in adverse event (AE) summaries. For long-term safety, all AEs from the first administration of adalimumab until week 52 and study completion were summarized.

All patients enrolled and treated with adalimumab were included in the efficacy analyses. Responses were assessed as no change/aggravated, improvement, marked improvement (MI), and complete remission (CR) using a composite efficacy index (Table 2), which combined global GI symptoms (Table 3) and endoscopic assessments. To analyze long-term efficacy, proportions of patients who achieved MI (both GI symptom score of $\leq 1$ [did not affect patient's daily life] and endoscopic score of $\leq 1$ [largest ulcer is $\leq 1 / 4$ origi-

Table 2. Evaluation Criteria for Global Gastrointestinal Symptoms

\begin{tabular}{|c|c|c|c|c|c|c|}
\hline & \multirow{3}{*}{ Endoscopic assessment } & \multicolumn{5}{|c|}{ Global GI symptoms assessment } \\
\hline & & 0 & 1 & 2 & 3 & 4 \\
\hline & & Free of symptoms & $\begin{array}{c}\text { Did not affect } \\
\text { patient's daily life }\end{array}$ & $\begin{array}{l}\text { Slightly affected } \\
\text { patient's daily life }\end{array}$ & $\begin{array}{l}\text { Affected patient's } \\
\text { daily life }\end{array}$ & $\begin{array}{l}\text { Critically affected } \\
\text { patient's daily life }\end{array}$ \\
\hline 0 & Complete ulcer healing & $\begin{array}{l}\text { Complete } \\
\text { remission }\end{array}$ & $\begin{array}{l}\text { Marked } \\
\text { improvement }\end{array}$ & Improvement & $\begin{array}{l}\text { No change or } \\
\text { aggravated }\end{array}$ & $\begin{array}{l}\text { No change or } \\
\text { aggravated }\end{array}$ \\
\hline 1 & $\begin{array}{l}\text { Largest ulcer is } \leq 1 / 4 \\
\text { original size }\end{array}$ & $\begin{array}{l}\text { Marked } \\
\text { improvement }\end{array}$ & $\begin{array}{l}\text { Marked } \\
\text { improvement }\end{array}$ & Improvement & $\begin{array}{l}\text { No change or } \\
\text { aggravated }\end{array}$ & $\begin{array}{l}\text { No change or } \\
\text { aggravated }\end{array}$ \\
\hline 2 & $\begin{array}{l}\text { Largest ulcer is between } 1 / 2 \\
\text { and } 1 / 4 \text { original size }\end{array}$ & Improvement & Improvement & Improvement & $\begin{array}{l}\text { No change or } \\
\text { aggravated }\end{array}$ & $\begin{array}{l}\text { No change or } \\
\text { aggravated }\end{array}$ \\
\hline 3 & $\begin{array}{l}\text { Largest ulcer still } \geq 1 / 2 \\
\text { original size, or expanded }\end{array}$ & $\begin{array}{c}\text { No change or } \\
\text { aggravated }\end{array}$ & $\begin{array}{c}\text { No change or } \\
\text { aggravated }\end{array}$ & $\begin{array}{c}\text { No change or } \\
\text { aggravated }\end{array}$ & $\begin{array}{c}\text { No change or } \\
\text { aggravated }\end{array}$ & $\begin{array}{l}\text { No change or } \\
\text { aggravated }\end{array}$ \\
\hline
\end{tabular}

At each assessment visit, gastrointestinal (GI) symptoms were evaluated over the course of the previous 2-week period. Patients who met the criteria for complete remission also met the criteria for marked improvement.

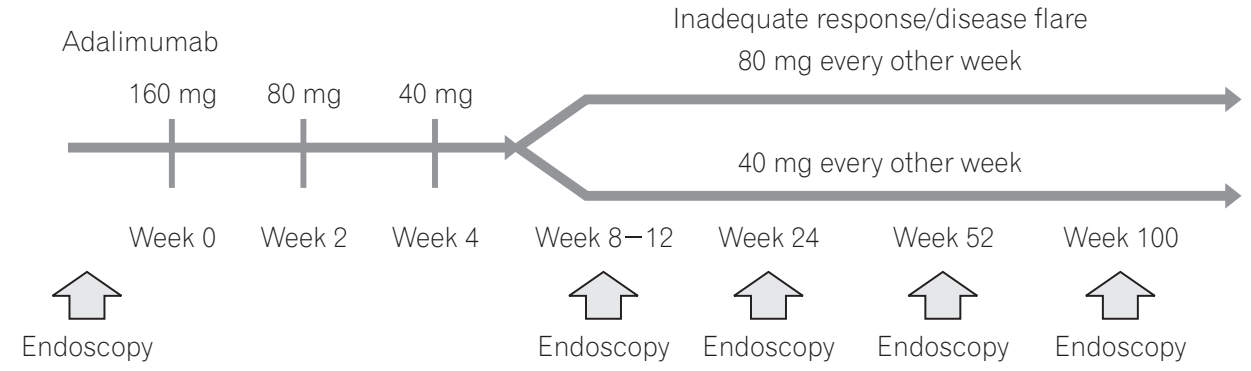

Fig. 1. Study design. 
Table 3. Evaluation of Gastrointestinal Symptoms

\begin{tabular}{cll}
\hline Score & \multicolumn{1}{c}{$\begin{array}{c}\text { Evaluation criteria for Gl symptoms and global } \\
\text { assessment of Gl symptoms }\end{array}$} & Interpretation \\
\hline 0 & Symptom free & Patient had no Gl symptoms. \\
1 & $\begin{array}{c}\text { Symptoms were present within the last } 2 \text { wk before assessment } \\
\text { but did not affect the subject's daily life. }\end{array}$ & $\begin{array}{c}\text { Patient had some Gl symptoms, but not severe enough to miss } \\
\text { school or work/skip housekeping. }\end{array}$ \\
2 & $\begin{array}{c}\text { Symptoms were present within the last } 2 \text { wk before assessment } \\
\text { and slightly affected the subject's daily life. }\end{array}$ & $\begin{array}{c}\text { Due to Gl symptoms, patient considered missing school or work/ } \\
\text { skip housekeeping, to be on the safe side. }\end{array}$ \\
& $\begin{array}{c}\text { Symptoms were present within the last 2 wk before assessment } \\
\text { and affected the subject's daily life. }\end{array}$ & $\begin{array}{c}\text { Due to Gl symptoms, patient seriously considered missing school } \\
\text { or work/skip housekeeping, but worked through the pain. }\end{array}$ \\
4 & $\begin{array}{c}\text { Symptoms were present within the last 2 wk before assessment } \\
\text { and critically affected the subject's daily life. }\end{array}$ & $\begin{array}{c}\text { Due to Gl symptoms, patient had no other choice to but to miss } \\
\text { school or work/skip housekeeping. }\end{array}$ \\
\hline
\end{tabular}

$\mathrm{Gl}$, gastrointestinal.

nal size]) at weeks 52 and 100 were calculated. MI included patients with CR, defined as a GI symptom score of 0 (symptom-free) and an endoscopic score of 0 (complete ulcer healing). Furthermore, proportions of patients who achieved CR and had GI symptom scores of 0 and $\leq 1$, GI symptom improvement of $\geq 1$ point, and endoscopic scores of 0 and $\leq 1$ at weeks 52 and 100 were calculated. The long-term efficacy was analyzed using nonresponder imputation (patients with missing data were considered nonresponders).

\section{RESULTS}

\section{Patients}

Twenty patients were enrolled and treated with adalimumab; 18 patients were evaluated at week 52 and 15 were still receiving adalimumab treatment at the time of study completion. Seven patients dose-escalated to adalimumab $80 \mathrm{mg}$ every other week. The mean $( \pm \mathrm{SD})$ duration of adalimumab treatment was $637.5( \pm 237.98)$ days. Patient demographics and baseline characteristics have already been reported. ${ }^{2}$ Infliximab (IFX) was administered to 3 patients $\geq 57$ days prior to baseline. Patients who did not respond to IFX were excluded from the study based on the exclusion criteria. In 1 patient, azathioprine was added to treat lower GI hemorrhage at week 85 . There were no patients who started corticosteroid for the first time after week 52. Three out of 18 patients discontinued the study after being evaluated at week 52 (1 patient with endoscopic healing and 2 patients without endoscopic healing at week 52). One patient discontinued because of AEs, 1 patient withdrew from the study due to the patient's private reason, and the other patient developed anti-adalimumab antibodies and had undetectable concentrations of adalimumab.

\section{Safety}

The incidence of AEs during the study was 544.4 events/100 person-years and was comparable to that at week 52 (560.4 events/100 person-years) (Table 4). The most common AEs during the study were nasopharyngitis (11/20 patients); headache (5/20 patients); and Behçet's syndrome, back pain, and cough (each $4 / 20$ patients). All AEs were those expected in patients treated with adalimumab or were anticipated in intestinal BD patients. No deaths occurred during the study, and no patient had malignancy, congestive heart failure, demyelination, or lupus-like syndrome. The incidence of serious AEs (25.8/100 person-years) and severe AEs especially serious infections during the study was higher than that at week 52 (5.5/100 person-years); 6 patients experienced 9 serious AEs (intestinal abscess/appendicitis/perforated appendicitis [these 3 events simultaneously occurred in 1 patient], Behçet's syndrome, autoimmune thyroiditis, intestinal obstruction, lower GI hemorrhage, subileus, and spondylitic myelopathy), of which only intestinal obstruction occurred before week 52. AEs leading to the discontinuation of the study drug occurred in 3 patients (Behçet's syndrome, intestinal obstruction and abscess intestinal/appendicitis/ appendicitis perforated). None of the laboratory results and vital signs showed clinically significant changes. All of the serious AEs were deemed unrelated or probably unrelated to the study drug, and the patients recovered during the treatment or after discontinuation, except those with Behçet's syndrome, which relapsed after an AE of appendicitis and discontinuation of adalimumab but resolved with medication and fasting.

Furthermore, 1 pregnancy was reported in 1 patient in this study. The 38 years old subject reported the pregnancy at the follow-up visit. She delivered a normal baby by cesarean 
Table 4. Adverse Events through Week 52 and through the Study

\begin{tabular}{|c|c|c|}
\hline \multirow{2}{*}{ Adverse events, (E/100 person-years) } & Through week $52(n=20)$ & Through the study $(n=20)$ \\
\hline & 18.2 person-years & 34.9 person-years \\
\hline Any adverse event & $102(560.4)$ & $190(544.4)$ \\
\hline At least possibly drug-related & $9(49.5)$ & $10(28.7)$ \\
\hline At least probably not drug-related & $41(225.3)$ & $58(166.2)$ \\
\hline Severe & 0 & $3(8.6)$ \\
\hline Serious & $1(5.5)$ & $9(25.8)$ \\
\hline Leading to discontinuation of study drug & $2(11.0)$ & $5(14.3)$ \\
\hline \multicolumn{3}{|l|}{ Adverse event of interest } \\
\hline Infection & $30(164.8)$ & $61(174.8)$ \\
\hline Serious infection & 0 & $3(8.6)$ \\
\hline Allergic reaction including angioedema/anaphylaxis & $2(11.0)$ & $5(14.3)$ \\
\hline Noncutaneous vasculitis ${ }^{\mathrm{a}}$ & $3(16.5)$ & $4(11.5)$ \\
\hline Hematologic disorders including pancytopenia & 0 & $3(8.6)$ \\
\hline Injection site reaction & $2(11.0)$ & $3(8.6)$ \\
\hline
\end{tabular}

Values are presented as number and incidence of adverse events.

a'Behçet's syndrome.

E, events.

\section{A}

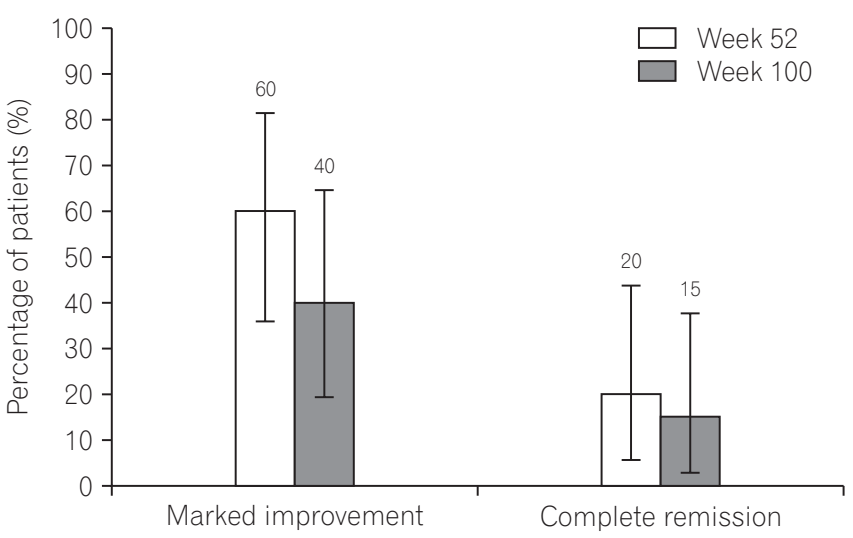

C

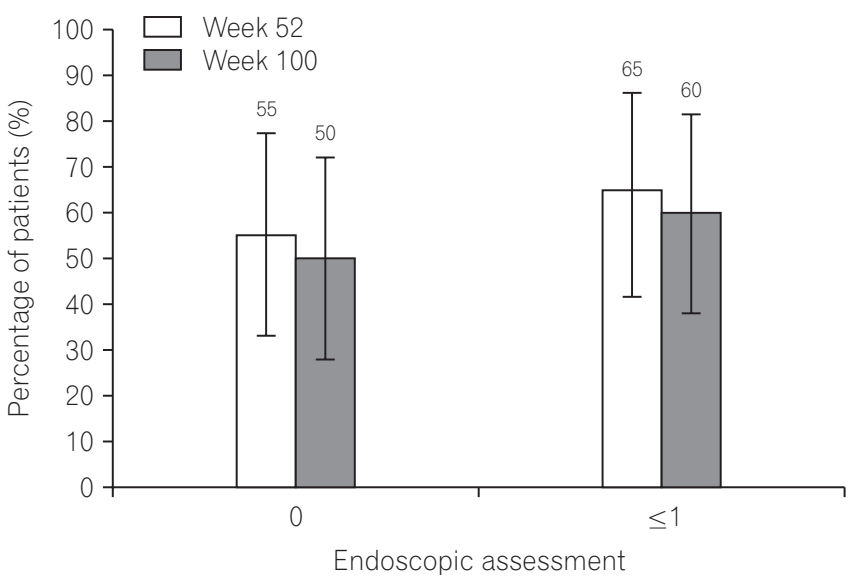

B

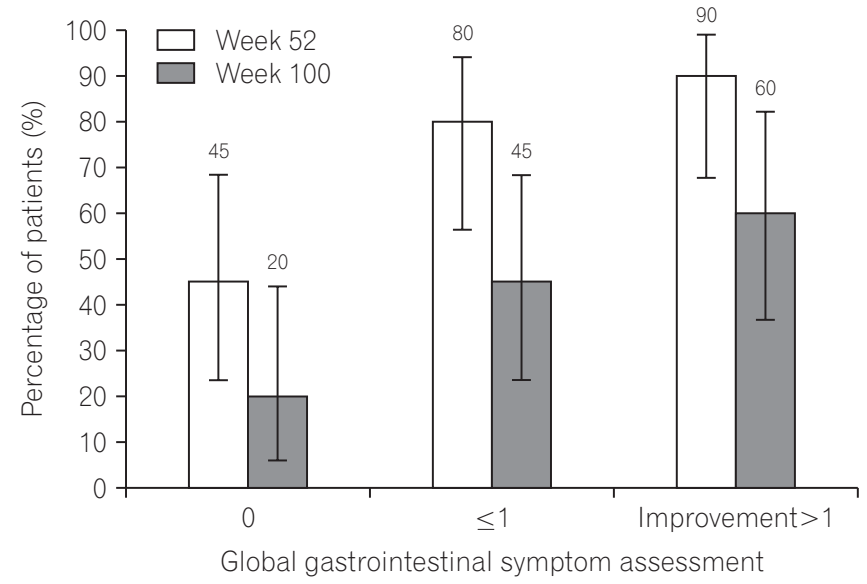

Fig. 2. Long-term efficacy as assessed by the composite efficacy index (A), global gastrointestinal symptom assessment (B), and endoscopic assessment (C). 
section at 39 years old.

\section{Efficacy}

In total, $60.0 \%$ of patients (12/20) at week 52 and $40.0 \%$ of patients $(8 / 20)$ at week 100 achieved MI; $20.0 \%$ of patients $(4 / 20)$ at week 52 and $15.0 \%$ of patients $(3 / 20)$ at week 100 (Fig. 2A) achieved CR. Ninety percent of patients (18/20) showed $\geq 1$ category of improvement in GI symptoms at week 52, this rate was largely sustained through week 100 $(60.0 \%, 12 / 20)$. For GI symptoms assessment specifically, the proportions of patients with scores $\leq 1$ were $80.0 \%(16 / 20)$ at week 52 and $45.0 \%(9 / 20)$ at week $100 ; 45.0 \%$ of patients $(9 / 20)$ at week 52 and $20.0 \%$ of patients $(4 / 20)$ at week 100 (Fig. 2B) had scores of 0 . The proportion of patients with an endoscopic improvement score of $\leq 1$ were $65.0 \%(13 / 20)$ at week 52 and $60.0 \%(12 / 20)$ at week 100; an endoscopic improvement score of 0 was observed in $55.0 \%$ of patients $(11 / 20)$ at week 52 and $50.0 \%$ of patients $(10 / 20)$ at week 100 (Fig. 2C). Fifteen subjects were assessable at week 100; 8 of 12 patients receiving concomitant corticosteroids at baseline tapered their dose, and 6 discontinued corticosteroids by week 100. Of these 6 patients, 3 achieved MI, but no patients experienced CR. One of the 3 patients who discontinued IFX $\geq 57$ days prior to baseline achieved MI at week 100 but another one did not, and the other one discontinued at week 10 without MI.

\section{DISCUSSION}

Long-term safety of adalimumab treatment in intestinal BD patients was shown in the 2-year clinical trial. While this study was limited in size due to the rarity of intestinal BD it is the longest and largest study with a biologic therapy reported to date. ${ }^{4}$ There were no unexpected trend and all serious AEs were considered unrelated or probably not related to the treatment. The rates of any $\mathrm{AE}$ and any $\mathrm{AE}$ at least possibly drug related did not increase from data observed through week 52 with increasing exposure. According to the safety results, long-term treatment with adalimumab in intestinal BD patients can be concluded to tolerate. The observed profile in this study did not identify any new safety signals and the safety profile for intestinal BD is similar to that in the approved indications. ${ }^{5}$

Half of the patients had plural ileocecal ulcers and $60 \%$ had $>2 \mathrm{~cm}$ in diameter at baseline, suggesting that moderate intestinal BD patients were enrolled in this study. Among patients with intestinal $\mathrm{BD}$, the surgical intervention rate re- mains high; $31 \%$ to $46 \%$ at 10 years after diagnosis, therefore to prevent surgery and reduce relapses, sustained remission should be the goal of medical therapy for intestinal BD. Therefore, data regarding the long-term safety and efficacy outcomes of medical interventions for patients with intestinal BD answer important clinical questions.

The overall incidence of AEs during the study was comparable to that at week 52 . While the incidence of serious and severe AEs with increased follow up than that at week 52, 8 serious AEs with onset after week 52 were observed in 3 patients, 6 of which affected the GI system. The increase in serious AEs after week 52 was considered to be related to intestinal BD worsening in these individuals. The incidence of AEs under long-term adalimumab treatment in this study was similar or slightly lower than that in patients with CD who had undergone up to 4 years of treatment with adalimumab. ${ }^{6,7}$ According to a long-term safety analysis that included 5 indications of adalimumab, serious infections were most frequent (1.4-6.7 events/100 person-years). ${ }^{4}$ The rate of serious infection in this study was 8.6 events/100 personyears; however, 3 events associated with appendicitis simultaneously occurred in 1 patient. Although no conclusion in terms of comparison of the $\mathrm{AE}$ incidences with longer-term treatment could be drawn from these results considering the small number of patients, long-term treatment with adalimumab was not associated with unexpected trend for the patient population in this study.

To date, corticosteroids and immunosuppressants have been empirically used for the treatment of intestinal BD., ${ }^{8,9}$ Anti-TNF- $\alpha$ agents were approved in Japan on the basis of the results from 52 -week clinical trials, ${ }^{2,4}$ but prior to this report the efficacy of these agents for longer-term treatment of intestinal BD was unknown. Retrospective analyses of intestinal BD patients after 2 years of pharmacotherapy (including corticosteroids, 5 -aminosalicylic acid, and colchicine) have indicated a $25 \%$ disease recurrence rate in Korean patients $^{10}(\mathrm{n}=93)$ and a $25 \%$ to $75 \%$ disease recurrence rate in nonsurgically $(\mathrm{n}=12)$ and surgically $(\mathrm{n}=8)$ treated Japanese patients. ${ }^{11}$ After 30 weeks of treatment with IFX, the rate of patients who achieved a CR was $54.5 \%(6 / 11) ;{ }^{12}$ evaluation of the effects of IFX beyond 30 weeks has not been reported. Similar trends were observed for MI, GI symptoms, and endoscopic assessment results. This long-term evaluation of the efficacy of adalimumab confirmed that the high rate of disease improvement at 52 weeks reported ${ }^{2}$ was sustained for 2 years. Prospective clinical trial evidence is important, as until now data regarding the outcome of anti-TNF treatment of patients with intestinal $\mathrm{BD}$ has been limited to retrospec- 
tive cohort studies of IFX treatment. ${ }^{13,14}$

In conclusion, it has been demonstrated that approximately 2 years of long-term treatment for intestinal BD with adalimumab was well tolerated without any unexpected AEs. It also found that the long-term adalimumab therapy helped achieve and sustain reduction of clinical and endoscopic disease activity in patients with intestinal BD refractory to conventional treatment. The recent approval for an additional indication of intestinal BD in Japan and other countries will allow large-scale data accumulation of adalimumab use in clinical practice and will contribute to a better understanding of the drug's characteristics in regard to the treatment of this patient population.

\section{ACKNOWLEDGEMENTS}

The authors thank Kae Uetani of Statcom Co., Ltd., Tokyo, Japan, for medical writing and editing services in the development of this manuscript. AbbVie GK provided funding to Statcom Co., Ltd. for this work. The authors also thank Joel Peterson, Pharm.D. of AbbVie Inc. for critical review of this work.

\section{REFERENCES}

1. Sakane T, Takeno M, Suzuki N, Inaba G. Behçet's disease. N Engl J Med 1999;341:1284-1291.

2. Tanida S, Inoue N, Kobayashi K, et al. Adalimumab for the treatment of Japanese patients with intestinal Behçet's disease. Clin Gastroenterol Hepatol 2015;13:940-948.e3.

3. The diagnostic criteria for Behçet's disease (2003). Ministry of Health, Labour and Welfare Web site. http://www.mhlw.go.jp/ file/06-Seisakujouhou-10900000-Kenkoukyoku/0000089968. pdf. Accessed April 4, 2017.

4. Hibi T, Hirohata S, Kikuchi H, et al. Infliximab therapy for intestinal, neurological, and vascular involvement in Behcet disease: efficacy, safety, and pharmacokinetics in a multicenter, prospective, open-label, single-arm phase 3 study. Medicine (Baltimore) 2016;95:e3863. doi: 10.1097/MD.0000000000003863.
5. Burmester GR, Panaccione R, Gordon KB, McIlraith MJ, Lacerda AP. Adalimumab: long-term safety in 23458 patients from global clinical trials in rheumatoid arthritis, juvenile idiopathic arthritis, ankylosing spondylitis, psoriatic arthritis, psoriasis and Crohn's disease. Ann Rheum Dis 2013;72:517-524.

6. Watanabe M, Hibi T, Mostafa NM, et al. Long-term safety and efficacy of adalimumab in Japanese patients with moderate to severe Crohn's disease. J Crohns Colitis 2014;8:1407-1416.

7. Panaccione R, Colombel JF, Sandborn WJ, et al. Adalimumab maintains remission of Crohn's disease after up to 4 years of treatment: data from CHARM and ADHERE. Aliment Pharmacol Ther 2013;38:1236-1247.

8. Hatemi G, Silman A, Bang D, et al. EULAR recommendations for the management of Behçet disease. Ann Rheum Dis 2008;67:1656-1662.

9. Hisamatsu T, Ueno F, Matsumoto T, et al. The 2nd edition of consensus statements for the diagnosis and management of intestinal Behçet's disease: indication of anti-TNFalpha monoclonal antibodies. J Gastroenterol 2014;49:156-162.

10. Chung MJ, Cheon JH, Kim SU, et al. Response rates to medical treatments and long-term clinical outcomes of nonsurgical patients with intestinal Behçet disease. J Clin Gastroenterol 2010;44:e116-e122. doi: 10.1097/MCG.0b013e3181c8a50f.

11. Naganuma M, Iwao Y, Inoue N, et al. Analysis of clinical course and long-term prognosis of surgical and nonsurgical patients with intestinal Behçet's disease. Am J Gastroenterol 2000;95:2848-2851.

12. REMICADE: package insert. Osaka: Mitsubishi Tanabe Pharma Corporation, 2017.

13. Lee JH, Cheon JH, Jeon SW, et al. Efficacy of infliximab in intestinal Behçet's disease: a Korean multicenter retrospective study. Inflamm Bowel Dis 2013;19:1833-1838.

14. Kinoshita H, Kunisaki R, Yamamoto H, et al. Efficacy of infliximab in patients with intestinal Behçet's disease refractory to conventional medication. Intern Med 2013;52:1855-1862. 